\title{
Effect of Magnetic Field on Blood Flow (Elastico- Viscous) Under Periodic Body Acceleration in Porous Medium
}

\author{
${ }^{1}$ Varun Mohan, ${ }^{2}$ Dr. Virendra Prasad, ${ }^{3}$ Dr.N.K.Varshney, \\ ${ }^{4}$ Dr. Pankaj Kumar Gupta \\ ${ }^{I}$ Department of Mathematics D.S Degree College Aligarh, ${ }^{2}$ Ex HOD of Deptt. Of Mathematics D.S. Degree \\ college Aligarh, ${ }^{3}$ Ret. Prof. S.V. College Aligarh, ${ }^{4}$ Aligarh College Of Engg. \& Tech. Aligarh.
}

\begin{abstract}
Aim of this study is to investigate the effect of magnetic field on blood flow in cylindrical artery through porous medium. In this paper blood is considered elastico viscous, Non Newtonian fluid and flow is assumed as fully developed and laminar. Laplace transforms and Finite Hankel Transforms are used to obtain the analytical expression for velocity profile, flow rate and fluid acceleration. The effect of magnetic field on velocity and fluid acceleration has been discussed with the help of graphs. It is found that velocity distribution, flow rate and fluid acceleration of blood in cylindrical artery decrease as magnetic field increases.
\end{abstract}

Keywords: - Elastico Viscous fluid, Laminar flow, porous medium, fully developed flow, Laplace transforms and Finite Hankel Transform

\section{Introduction}

The study of magneto hydro dynamics flows through porous medium has been studied by many authors. Liquid carriers as magnetic particles suspended in flowing blood serves as drug carriers of serious diseases to the diseased site. This type of energy affects biological processes not through heat production but through electrically induced charges in the environment of the cell within the organisms. It is known from magneto hydro dynamics that when magnetic field is applied to a moving electrically conducting fluid electrical currents are induced in the fluid. The interaction between induced currents and magnetic field produces Lorentz forces as an external force which retards blood flow.

In recent years the flow of fluids through porous media has become the field of attention for many authors as the study of flow of an electrically conducting fluid has many applications in medical sciences such as MHD. G. Ahmadi and R. Manvi,1971, [1] discussed the equations of motion for viscous flow through porous medium. R.K. Dash,K.N. Mehta and G. Jayarman,2006 [2] . Considered Casson fluid flow in a pipe. R. Ponalgusammy,2007 [3] studied blood flow through an artery with mild stenosis. P. Nagarani and G. Sarojamma,2008 [4] mentioned the effect of body acceleration on pulsatile flow of casson fluid through a mild stenosed artery in his work. Devajyoti Biswas et. Al. (2009) [5] studied the body acceleration in a constricted artery. Sanjeev Kumar et. Al. (2010)[6] developed the mathematical model of effect of body acceleration on blood flow in time dependent stenosed artery. J.C. Mishra et. Al. (2011)[7] investigated the behaviour of blood flow in artery under the effect of external magnetic field. N.K Varshney and Raja Agrawal (2011) [8] studied MHD flow of couple stress fluid through an inclined circular tube with periodic body acceleration. Anil Kumar et. Al.(2012)[9] suggested a model of blood flow in elastico viscous fluid under periodic body acceleration with porous effect. In the present study the effect of magnetic field on blood flow (elastico viscous) under periodic body acceleration in porous medium has been studied. This work is very useful in understating the behaviour of blood flow in porous medium.

\section{Mathematical model:-}

In the present investigation we assume the motion of blood as an incompressible fluid in the presence of body acceleration along the radius of circular tube and also consider the flow as axially symmetric. The pressure gradient is given by

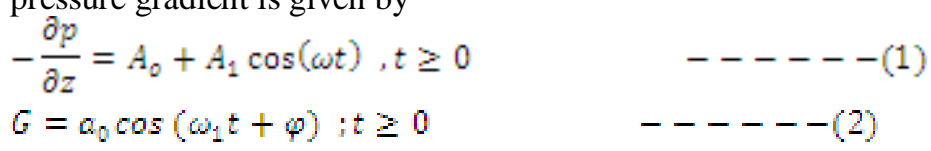

Where $A_{0}$ is the steady state part of pressure gradient, $A_{1_{\Omega}}$ the amplitude of the oscillatory part; $\omega=2 \pi f$ and $f$ the heart pulse frequency; $a_{0}$ the amplitude of body acceleration ; $\omega_{1}=2 \pi f_{1}$ and $f_{1}$ is body acceleration; $\varphi$ is phase difference; $\mathrm{z}$ axial direction. 


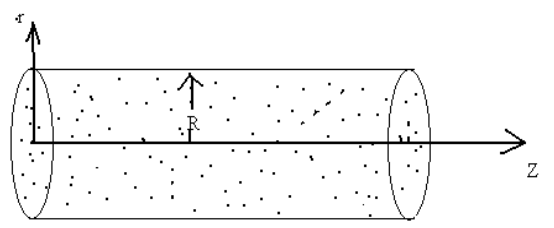

figure-1 geometriy diagram of blood flow

through artery

$\rho \frac{\partial u}{\partial t}=A_{0}+A_{1} \cos (\omega t)+\alpha_{0} \cos \left(\omega_{1} t+\varphi\right)+\left(\mu+\mu_{1} \frac{\partial}{\partial t}\right)\left(\frac{\partial^{2} u}{\partial r^{2}}+\frac{1}{r} \frac{\partial u}{\partial r}\right)-K u-\sigma B_{0}^{2} u-$

Where $\mathrm{u}(\mathrm{r}, \mathrm{t})$ is the velocity in axial direction, $\rho$ and $\mu$ are the density and viscosity of blood. $\mu_{1}$ is elastic viscosity coefficient of blood, $\sigma$ is the electrical conductivity, $\mathrm{K}$ the porous effect and $\mathrm{r}$ is radial coordinate. Let us introduce following dimensionless quantities as floows:

$u^{*}=\frac{u}{\omega R}, r^{*}=\frac{r}{R}, A_{0}^{*}=\frac{R}{\omega \mu} A_{0} A_{1}^{*}=\frac{R}{\omega \mu} A_{\mathbb{1}_{0}} z^{*}=\frac{z}{R}, \quad t^{*}=t \omega_{v} \quad a_{0}^{*}=\frac{a_{0}}{\mu \omega}--(4)$

On dropping the stars equation in (3) becomes

$\alpha^{2} \frac{\partial u}{\partial t}=A_{0}+A_{1} \cos (t)+a_{0} \cos (b t+\varphi)+\left(1+\beta \frac{\partial}{\partial t}\right)\left(\frac{\partial^{2} u}{\partial r^{2}}+\frac{1}{r} \frac{\partial u}{\partial r}\right)-\left(H^{2}+M^{2}\right) u--(5)$

$\beta=\left(\frac{\omega \mu_{1}}{\mu}\right)$ is dimensionless parameter governing elastic-viscosity of the fluid, $\alpha=\mathbb{R} \sqrt{\left(\frac{\omega \rho}{q}\right)}$ is Womersley parameter; $H=R \sqrt{\left(\frac{R}{R}\right)}$ is permeability parameter; $M=R B_{\mathbb{Q}} \sqrt{\frac{\sigma}{G K}}$ is Hartmann number; $b=\left(\frac{\omega}{\omega_{1}}\right)$ and the radius of the tube.

The initial and boundary conditions are

$u\left(r_{0}, 0\right)=\frac{A_{0}+A_{1}}{H^{2}+M^{2}}\left(-\left(\frac{I_{0}\left(\sqrt{\left(H^{2}+M^{2}\right)}\right)}{I_{0}\left(\sqrt{\left(H^{2}+M^{2}\right)}\right)}\right)\right)--(6)$

$u(1, t)=0$------- $(7)$

$\mathrm{u}(0, \mathrm{t})$ is finite

If $\mathrm{f}(\mathrm{r})$ satisfies the Dirichlet's conditions in the closed interval $(0,1)$ then finite Hankel transform is given by $f^{*}\left(\lambda_{\mathrm{n}}\right)=\int_{0}^{1} r f(r) J_{0}\left(r \lambda_{\mathrm{n}}\right) d r \quad-\cdots-(9)$

Where $\lambda_{\mathrm{n}}$ are the roots of equation $\mathrm{J}_{0}(\mathrm{r})=0$ and

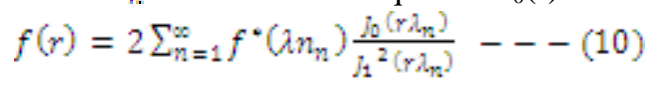

\section{Numerical Method}

By using Laplace transform (5) becomes

$\alpha^{2} s \bar{u}-\alpha^{2} u(r, 0)$

$$
\begin{aligned}
& =\frac{A_{0}}{s}+\frac{A_{1} s}{s^{2}+1}+\frac{a_{0}(s \cos \varphi-b \sin \varphi)}{s^{2}+b^{2}}+\left(\frac{\partial^{2}}{\partial r^{2}}+\frac{1}{r} \frac{\partial}{\partial r}\right)\left(\bar{u}+\beta s \bar{u}-\beta u\left(r_{v} 0\right)\right. \\
& -\left(H^{2}+M^{2}\right) \bar{u}----(11)
\end{aligned}
$$

On applying the finite Hankel transform equation on equation (11) in the light of (7), we obtain

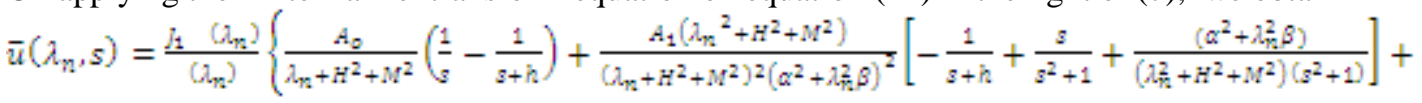

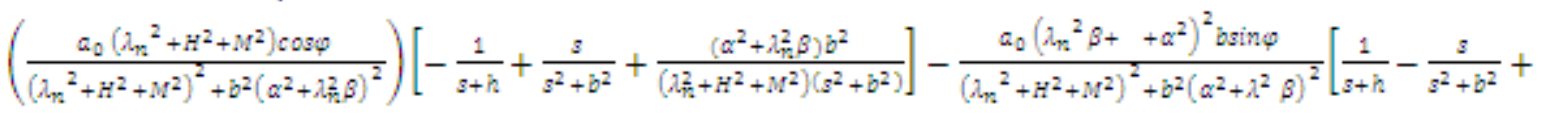

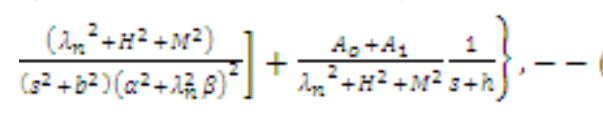

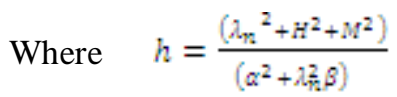

The Laplace and Hankel inversion of equation (12) gives the final solution as given below. 


$$
\begin{aligned}
& u(r, t)=2 \sum_{n=1}^{\infty} \frac{J_{0}\left(r \lambda_{n}\right)}{\lambda_{n} j_{1}\left(\lambda_{n}\right)}\left\{\frac{A_{0}}{\left(\lambda_{n}{ }^{2}+H^{2}+M^{2}\right.}+\frac{A_{1}\left[\left(\lambda_{n}{ }^{2}+H^{2}+M^{2}\right) \cos t+\left(\alpha^{2}+\lambda_{n B}^{2} \beta\right) \sin t\right]}{\left(\lambda_{n}{ }^{2}+H^{2}+M^{2}\right)^{2}+\left(\alpha^{2}+\lambda^{2} \beta\right)^{2}}\right. \\
& +\frac{a_{0}\left[\left(\lambda_{n}{ }^{2}+H^{2}+M^{2}\right) \cos (b t+\varphi)+\left(\alpha^{2}+\lambda_{n}^{2} \beta\right) \sin (b t+\varphi]\right.}{\left(\lambda_{n}^{2}+H^{2}+M^{2}\right)^{2}+\left(\alpha^{2}+\lambda^{2} \beta\right)^{2}} \\
& +e^{-h t}\left[\frac{A_{0}}{\lambda_{n}{ }^{2}+H^{2}+M^{2}}+\frac{A_{1}\left[\left(\lambda_{n}{ }^{2}+H^{2}+M^{2}\right)\right]}{\left(\lambda_{n 2}{ }^{2}+H^{2}+M^{2}\right)^{2}+\left(\alpha^{2}+\lambda^{2} \beta\right)^{2}}-\frac{A_{0}+A_{1}}{\lambda_{n}{ }^{2}+H^{2}+M^{2}}\right] \\
& \left.+\frac{a_{0}\left[\left(\lambda_{s 2}^{2}+H^{2}+M^{2}\right) \cos \varphi+\left(\alpha^{2}+\lambda_{s 1}^{2} \beta\right) \sin \varphi\right]}{\left(\lambda_{n 2}^{2}+H^{2}+M^{2}\right)^{2}+b^{2}\left(\alpha^{2}+\lambda^{2} \beta\right)^{2}}\right\}
\end{aligned}
$$

The expression for the flow rate Q can be written as given below:

$$
\begin{aligned}
& Q=2 \int_{0}^{1} r u d r
\end{aligned}
$$

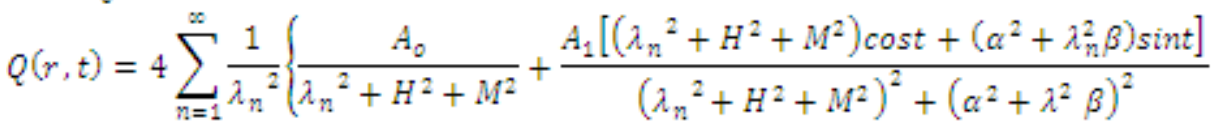

$$
\begin{aligned}
& +\frac{a_{0}\left[\left(\lambda_{n 2}^{2}+H^{2}+M^{2}\right) \cos (b t+\varphi)+\left(\alpha^{2}+\lambda_{n}^{2} \beta\right) \sin (b t+\varphi]\right.}{\left(\lambda_{n}^{2}+H^{2}+M^{2}\right)^{2}+\left(\alpha^{2}+\lambda^{2} \beta\right)^{2}} \\
& +e^{-\lambda t}\left[\frac{A_{0}}{\lambda_{n}{ }^{2}+H^{2}+M^{2}}+\frac{A_{1}\left[\left(\lambda_{n}{ }^{2}+H^{2}+M^{2}\right)\right]}{\left(\lambda_{n}{ }^{2}+H^{2}+M^{2}\right)^{2}+\left(\alpha^{2}+\lambda^{2} \beta\right)^{2}}-\frac{A_{0}+A_{1}}{\lambda_{n}{ }^{2}+H^{2}+M^{2}}\right] \\
& \left.+\frac{a_{0}\left[\left(\lambda_{n 2}{ }^{2}+H^{2}+M^{2}\right) \cos \varphi+\left(\alpha^{2}+\lambda_{n 1}^{2} \beta\right) \sin \varphi\right]}{\left(\lambda_{n 3}^{2}+H^{2}+M^{2}\right)^{2}+b^{2}\left(\alpha^{2}+\lambda^{2} \beta\right)^{2}}\right)
\end{aligned}
$$

Similarly the expression for fluid acceleration F can be obtained from

$F(r, t)=\frac{\partial u}{\partial t}$

$F(r, t)=$

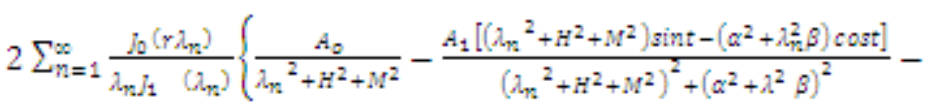

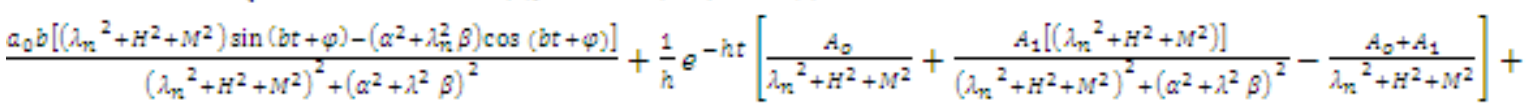

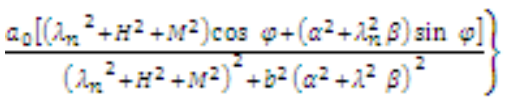

\section{Numerical Results and discussion:-}

Tthe expression for velocity $\mathrm{u}(\mathrm{r}, \mathrm{t}), \mathrm{f}(\mathrm{r}, \mathrm{t})$ and $\mathrm{Q}(\mathrm{r}, \mathrm{t})$ are obtained in equation (14),(16),(18) have been depicted In figures 2-3 by plotting $r$ versus $u$ for different values of Hartmann number $M$, syeady part of pressure gradient $A_{0}$, figure -4 is constructed for $r$ versus $f(r, t)$ for different values of $M$ and figure -5 is constructed for $t$ versus $\mathrm{Q}(\mathrm{r}, \mathrm{t})$ for different values of $\mathrm{M}$.

Figure 2 shows that $\mathrm{u}$ decreases with increase in $\mathrm{M}$, figure 3 shows that $\mathrm{u}$ decreases with increase in $\mathrm{A}_{0}$. Figure 4 indicates that there occurs a retardation in fluid velocity $\mathrm{u}(\mathrm{r}, \mathrm{t})$ and the amount of this retardation is decreased with the increase in M. Figure 5 demonstrated that flow rate $Q(r, t)$ decreases with increase in Hartmann number M.

\section{Conclusion:-}

It is clear from the above results and discussions that magnetic field effects largely on the axial velocity $\mathrm{u}(\mathrm{r}, \mathrm{t})$ and other parameters like $\mathrm{Q}(\mathrm{r} . \mathrm{t})$ and $\mathrm{f}(\mathrm{r}, \mathrm{t})$. From the figures 2-5 the amount of deviation of axial velocity, fluid acceleration and flow rate due to $M$ is shown. So by taking appropriate values of $M$ and other parameters we can control the axial velocity of blood in arteries. 
The present model gives a most general form of velocity expression involving $\mathrm{M}$ and $\mathrm{H}$ from which other mathematical models can easily be developed by considering other effects and parameters.

Here from all above discussion we can conclude that a careful choice of the values of the parameters of body acceleration, H,M, will effect the flow characteristics and hence can be used for better understanding of blood circulation in human body.

\section{References:-}

[1] G. Ahmadi and R. Manvi, "Equation of motion for viscous flow through a regis porous medium" Indian J. Tech. 9, 1971 , PP441-444.

[2] R.K. Dash,K.N. Mehta and G. Jayarman . "Casson fluid flow in a pipe filled with a homogeneous porous medium.” Int. J. Eng. Sci. 34, 2006, PP 1145-1156.

[3] R. Ponalgusammy,"Blood flow through an artery with mild stenosis." A two layered model, different shapes of stenosis and slip velocity at the wall." Journal of applied science, Vol.7 (2007) PP 1071-1077

[4] P. Nagarani and G. Sarojamma, "Effect of body acceleration on pulsatile flow of casson fluid through a mild stenosed artery." KoreaAustralia Rheology Journal volume -20, (2008). PP189-196.

[5] Devajyoty Biswas and Uday Shankar Chakraborty, "Pulsatile flow of blood in a constricted artery with body acceleration." AAM vol.4 No. 2 December 2009 PP 329-342.

[6] Sanjeev Kumar and Archana Dixit, "Mathematical model for the effect of body acceleration on blood flow in time dependent stenosed artery." International Journal of stability and fluid mechanics. Jan-2010 Vol. 1 No.-1 PP 103-115.

[7] J.C. Mishra, A. Sinha and G.C. Shit," Mathematical modelling of blood flow in a porous vessel having double stenosis in the presence of magnetic field.", international journal of biomathematics. June 2011 Vol 4 No.2 pp 207-225.

[8] N.K. Varshney and Raja Agarwal, "MHD pulsatile flow of couple stress fluid through an inclined circular tube with periodic body acceleration." Journal purvanchal academy of sciences, 2011 vol 17 pp 277-293.

[9] Anil Kumar, C.L. Varshney, Veer Pal Singh, "Mathematical modelling of blood flow in elastic viscus fluid under periodic body acceleration with porous effect." IJMR vol 1 issue 03 sept. 2012.

Figure 2:-

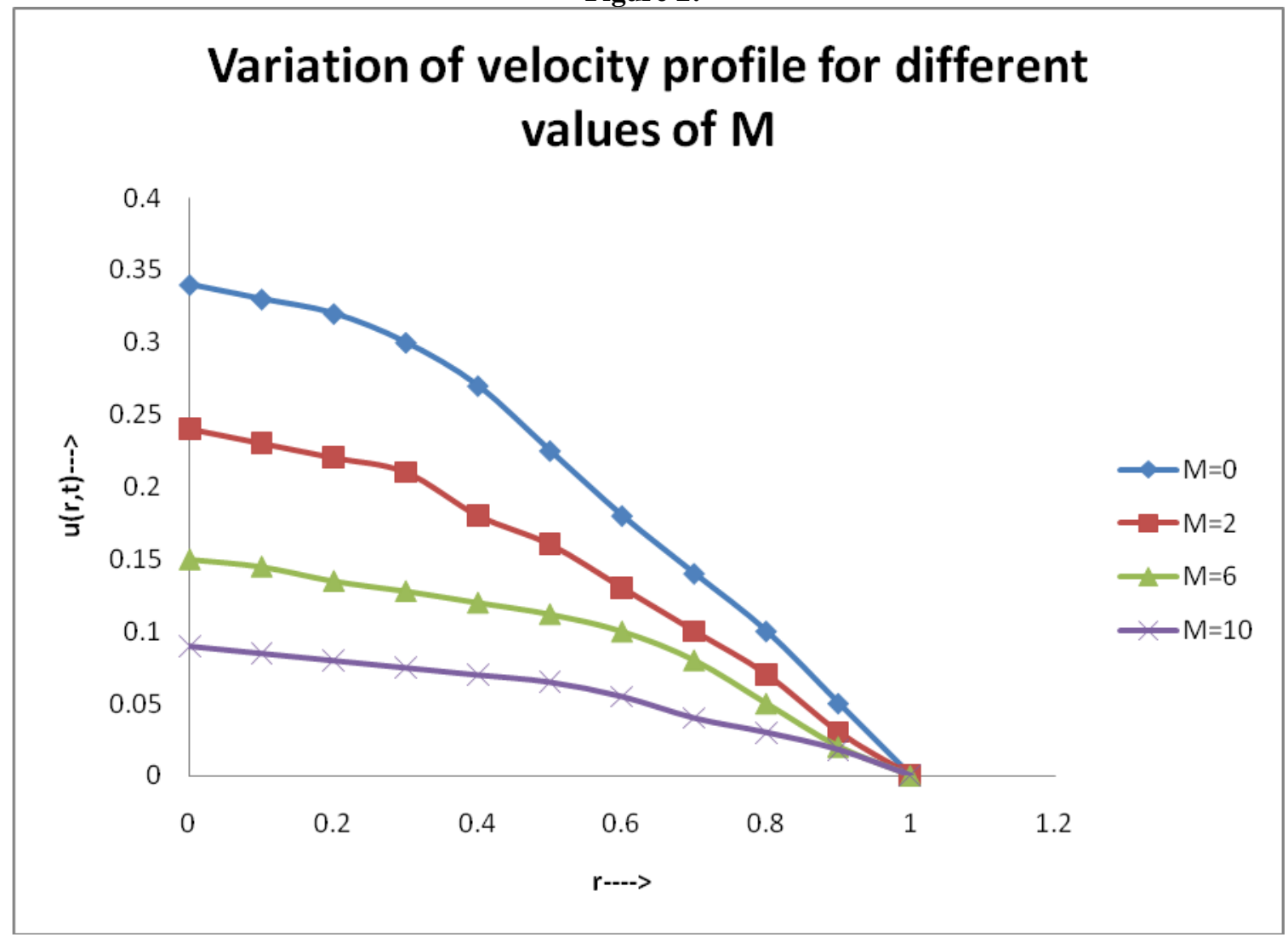

Taking $\mathrm{a}_{0}=3, \mathrm{~A}_{0}=2, \mathrm{~A}_{1}=4, \varphi=15^{\circledR}, \mathrm{H}=2.5, \mathrm{t}=.5, \mathrm{~b}=1, c=1, \bar{c}=1$

Figure-3 


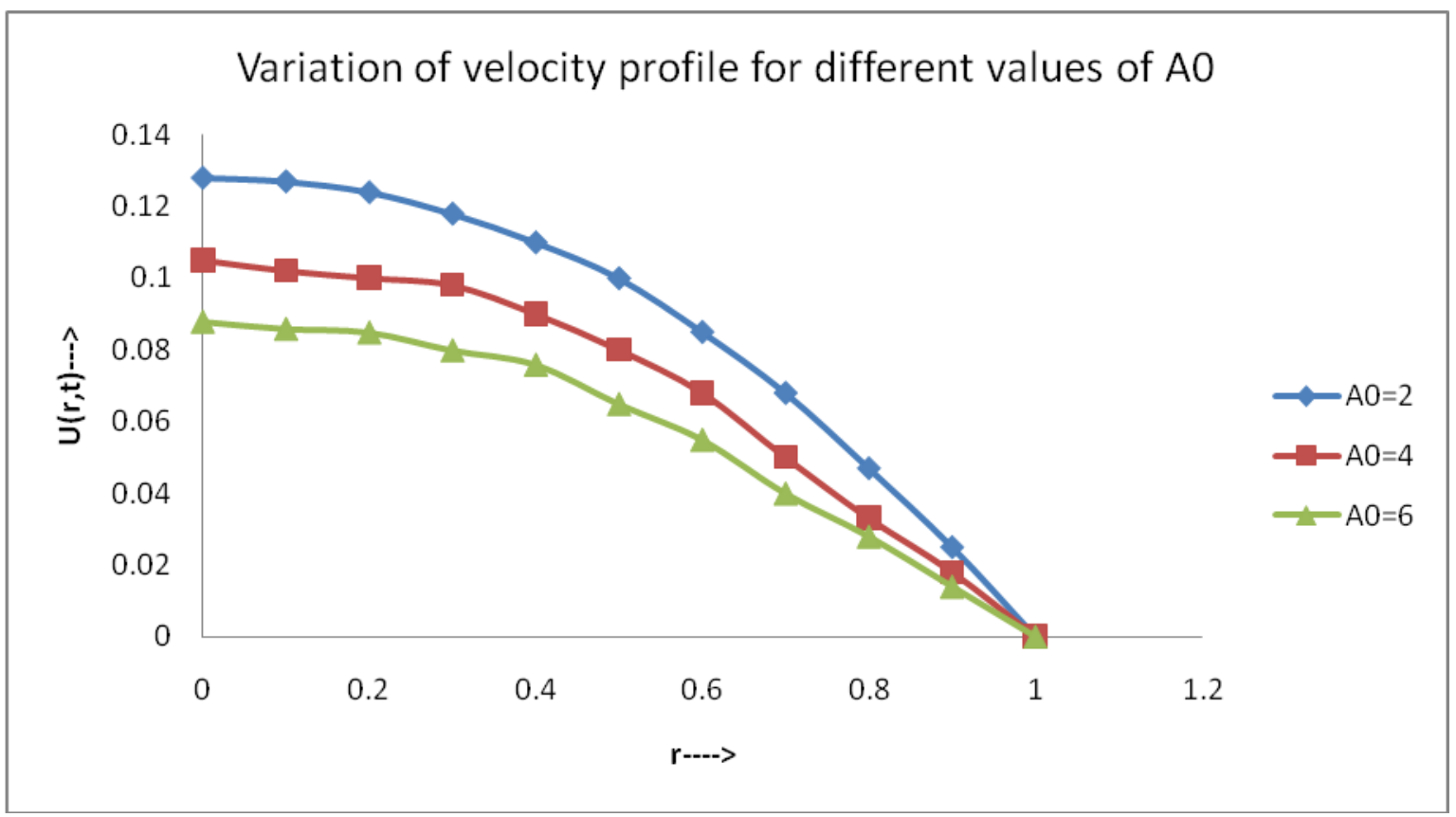

Taking $\mathrm{a}_{0}=3, \mathrm{~A}_{1}=4, \varphi=15^{\mathbb{0}}, \mathrm{H}=2.5, \mathrm{t}=.5, \mathrm{~b}=1, \alpha=1, \bar{\alpha}=1$

Figure-4

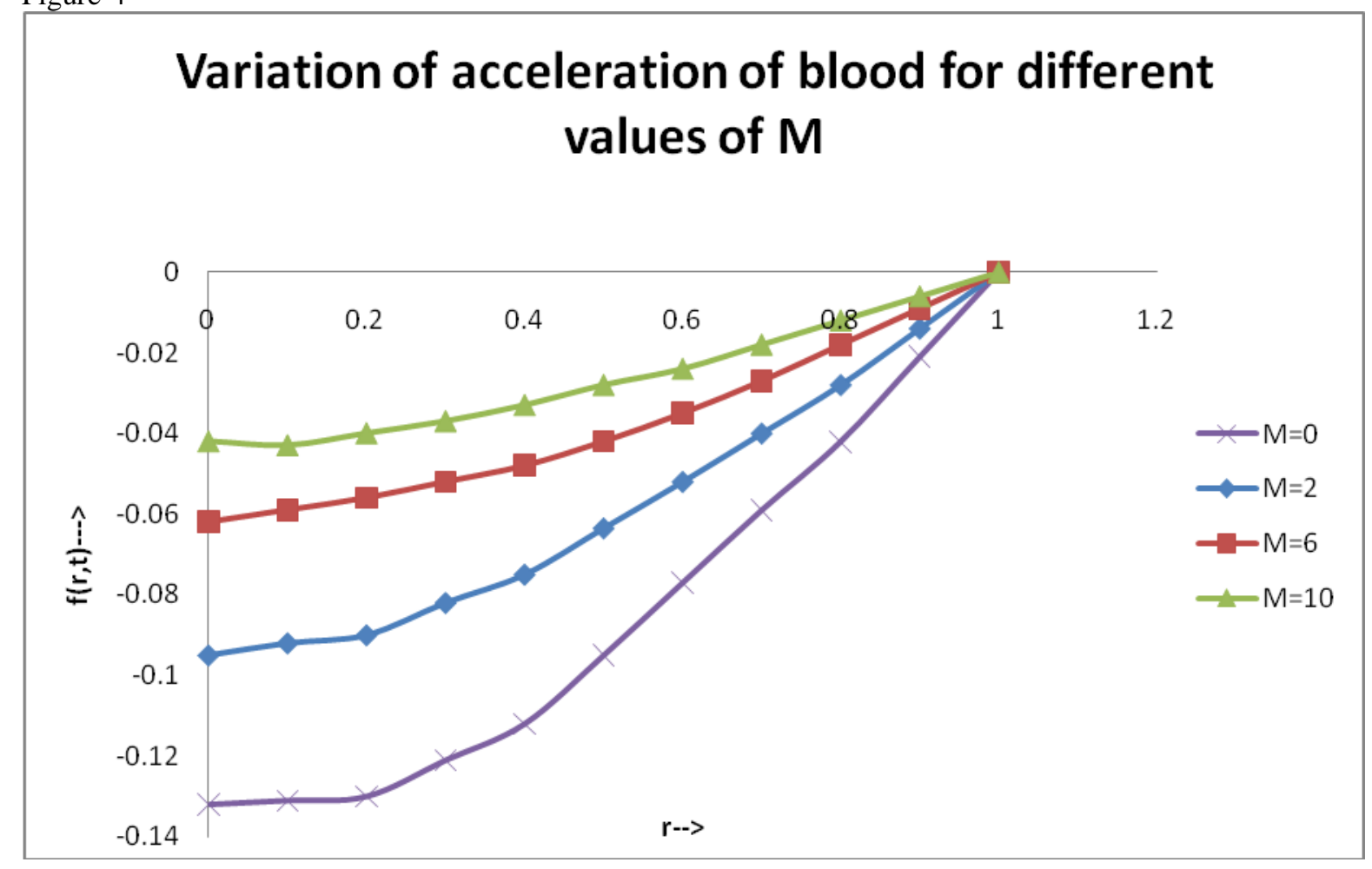

Taking $\mathrm{a}_{0}=3, \mathrm{~A}_{0}=2, \mathrm{~A}_{1}=4, \varphi=15^{\mathrm{Q}}, \mathrm{H}=2.5, \mathrm{t}=.5, \mathrm{~b}=1, \omega=1, \bar{\alpha}=1$ 
Figure-5:-

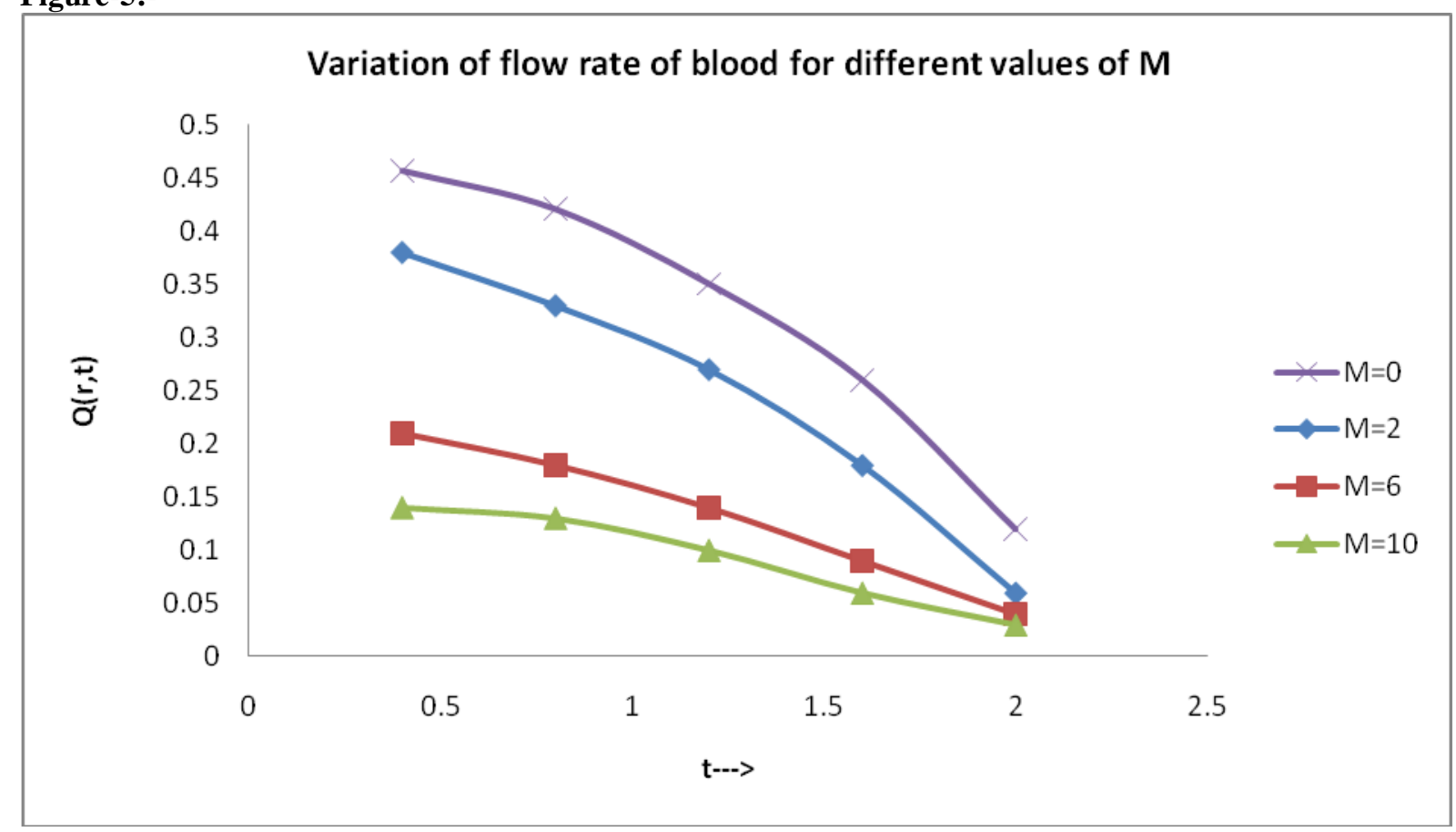

Taking $\mathrm{a}_{0}=3 \mathrm{~A} 0=2.0, \mathrm{~A} 1=4.0, \mathrm{~b}=1, \varphi=150, \bar{\kappa}=1, \alpha=1$ 